\title{
Amostragem para a estimativa de produção de sementes de castanheira-do-brasil em floresta nativa
}

Helio Tonini(1)

(1)Embrapa Agrosilvipastoril, Rodovia dos Pioneiros, MT-222, Km 2,5, CEP 785-50970 Sinop, MT. E-mail: helio.tonini@embrapa.br

Resumo - O objetivo deste trabalho foi determinar o tamanho necessário da amostra para estimar a produção individual e populacional de árvores de castanheira-do-brasil (Bertholletia excelsa) em floresta nativa, bem como avaliar diferentes sistemas de amostragem. Os dados foram obtidos em um castanhal na região do Itã, em Caracaraí, RR. A produção de frutos e sementes de 239 árvores foi monitorada de 2007 a 2010 . Com base na relação das características das árvores (diâmetro, tipo morfológico e variáveis de copa) com a produção, o tipo de alocação e a periodicidade da amostra, foram testados sete procedimentos de amostragem. Para verificar a precisão e a acurácia, foram tomadas 128 amostras da população e calculados o erro-padrão da média, o intervalo de confiança e o erro absoluto. Para estimar a produção de uma árvore a $1 \%$ de probabilidade, é necessário amostrar, em média, $97 \%$ dos frutos. Para o limite de erro de 10\%, é necessário amostrar 33\% dos frutos. A amostragem contínua, estratificada pelo diâmetro à altura do peito em seis estratos, e a alocação proporcional ao número de árvores na classe diamétrica são os procedimentos de amostragem mais indicados.

Termos para indexação: Bertholletia excelsa, floresta nativa, inventário florestal, produtos florestais não madeireiros.

\section{Sampling for the estimate of Brazil nut production in native forest}

\begin{abstract}
The objective of this work was to determine the necessary sample size in order to estimate individual and populational production of Brazil nut trees (Bertholletia excelsa) in a native forest, and to evaluate different sampling systems. Data were obtained from a grove in the Itã region, Caracaraí county, RR, Brazil. Fruit and seed production from 239 trees was monitored from 2007 to 2010. Seven sampling procedures were tested based on the relationship of tree characteristics (diameter, morphological type, and canopy traits) with production, allocation type, and sample periodicity. To verify the precision and accuracy, 128 samples were taken from population, then it was calculated the standard error of mean, confidence interval, and the absolute error. To estimate tree production at $1 \%$ of probability, it is necessary to sample $97 \%$ of fruit, on average. For a $10 \%$ error limit, it is necessary to sample $33 \%$ of fruit. Continuous sampling, stratified by the diameter at breast height in six strata, and proportional allocation to the number of trees in the diameter class are the most suitable sampling procedures.
\end{abstract}

Index terms: Bertholletia excelsa, native forest, forest inventory, nontimber forest products.

\section{Introdução}

As florestas tropicais mantêm grande parte da biodiversidade do planeta, e uma das principais estratégias para conservá-las é a exploração sustentável dos produtos florestais não madeireiros (PFNM). No entanto, o desenvolvimento e a implementação de sistemas de exploração sustentáveis, para a maioria dos PFNM, ainda carecem de informações referentes à sustentabilidade biológica, econômica e social (Boot, 1997).

São escassos os estudos que procuraram definir sistemas amostrais para inventários florísticos e madeireiros em florestas nativas na Amazônia (Higgins
\& Ruokolainen, 2004; Cavalcanti et al., 2009; Ubialli et al., 2009). Ainda mais raros, são os que estudaram o tema sobre produtos florestais não madeireros, como o de Farias (2012), que procurou definir o tamanho de parcelas para a estimativa do estoque de múltiplas espécies não madeireiras, em Manaus, AM. Segundo Wong et al. (2001), além de escassas, as pesquisas em inventário de PFNM são de pouca consistência estatística. Os problemas encontrados por pesquisadores que trabalham com inventário de PFNM referem-se às dificuldades com a aplicação da experimentação florestal tradicional, à falta de sistemas amostrais testados e validados e às poucas técnicas de mensuração disponíveis. 
Características comuns a diversas espécies que produzem PFNM, como raridade, agrupamento, sazonalidade, mobilidade e necessidade de quantificação por métodos não destrutivos, dificultam a utilização de técnicas tradicionais de inventário florestal (Wong et al., 2001). Além disso, deve-se evitar o emprego de sistemas amostrais dispendiosos, que podem colocar em risco o retorno financeiro da atividade não madeireira, pois a densidade e a disponibilidade dos PFNM geralmente são menores do que a da madeira, e o tempo e o custo requeridos para estimar os parâmetros com razoável grau de acurácia podem ser impraticáveis (Evans \& Viengkham, 2001).

Entre as espécies tropicais que produzem amêndoas, a castanheira-do-brasil (Bertholletia excelsa, H.B.K, Lecytidaceae) é a mais conhecida e solidamente estabelecida nos mercados doméstico e de exportação. Sua coleta ainda é realizada quase exclusivamente em florestas tropicais primárias (Peres et al., 2003; Salomão, 2009), o que a torna uma espécie-chave para o desenvolvimento socioeconômico das comunidades e a manutenção dos benefícios diretos e indiretos da floresta.

A quantificação da produção no tempo e de sua variação por árvore, entre populações e indivíduos, é fundamental para se obter uma estimativa adequada da produtividade potencial da castanheira-do-brasil. Zuidema \& Boot (2002), Wadt et al. (2005), Kainer et al. (2007) e Ivanov (2011) procuraram quantificar, estabelecer relações e modelar a produção de frutos e sementes de castanheira-do-brasil em diferentes regiões da Amazônia. No entanto, a definição do local de instalação, das dimensões e formas das unidades amostrais, da seleção das árvores e do número de frutos amostrados por população e indivíduo tem sido feita de forma arbitrária, com pouca fundamentação em estudos de amostragem.

Uma parte fundamental de um plano de manejo florestal sustentável é a elaboração do mapa de localização ou do censo das espécies que serão manejadas, que é realizado com o objetivo de se obter uma estimativa mais confiável da produção e o rastreamento da origem dos produtos, a fim de dar maior transparência à atividade e facilitar a vistoria de técnicos de órgãos ambientais e de instituições certificadoras (Alechandre et al., 2007). Porém, monitorar a produção de todos os indivíduos na área de manejo, na maioria dos casos, é inviável em razão dos recursos e do tempo disponíveis, o que torna necessário o desenvolvimento de processos de amostragem precisos, representativos e viáveis economicamente.

O objetivo deste trabalho foi determinar o tamanho necessário da amostra para estimar a produção individual e populacional de árvores de castanheira-do-brasil (Bertholletia excelsa) em floresta nativa, bem como avaliar diferentes sistemas de amostragem.

\section{Material e Métodos}

Os dados foram obtidos de um castanhal localizado na Fazenda Pau-Rainha, na região do Itã, Município de Caracaraí, RR, a $1^{\circ} 59^{\prime} 35^{\prime \prime} \mathrm{N}$ e $61^{\circ} 00^{\prime} 60^{\prime \prime} \mathrm{W}$. Nesta população, em uma área de 18 hectares, foi monitorada a produção de frutos e sementes de 239 árvores, de 2007 a 2010.

Todos os frutos das 239 árvores foram contados, e as sementes foram pesadas em balança de gancho digital com precisão de $50 \mathrm{~g}$, em duas ocasiões, durante o período de queda dos frutos que, nesta região da Amazônia, ocorre predominantemente de fevereiro a julho (Tonini, 2011). O diâmetro à altura do peito (DAP) limite de inclusão no inventário foi de $10 \mathrm{~cm}$; porém, para a avaliação dos diferentes processos de amostragem, elevou-se este diâmetro para $50 \mathrm{~cm}$, por ser considerado como o limite a partir do qual as árvores alcançam o dossel, expandem suas copas e atingem a maturidade de produção (Wadt et al., 2005; Kainer et al., 2007). Estes dados foram considerados como parâmetros para a população. Além do DAP, também foram determinadas a forma e posição da copa, segundo Synnott (1979), e o tipo morfológico: castanheiras rosas e brancas. Estes tipos morfológicos diferenciam-se pela cor da madeira, pelo potencial produtivo, pelo aspecto e porte das árvores e pela morfologia e tamanho dos frutos e sementes (Braga, 2007). No presente estudo, esta classificação foi realizada com base na experiência prática dos extrativistas.

Para calcular o tamanho da amostra e estimar a produção de frutos por árvore, foram amostrados 513 frutos produzidos por oito árvores, no ano de 2010. Os frutos foram coletados em campo, e abertos e medidos em laboratório. A medição do diâmetro do fruto $(\mathrm{cm})$ foi realizada em duas posições centrais, tomadas de forma perpendicular, com paquímetro digital. Todas as sementes foram contadas e pesadas em 
balança digital com precisão de $0,1 \mathrm{~g}$. Determinou-se a correlação de Pearson entre o diâmetro dos frutos e o número e peso de sementes. A intensidade de amostragem, ou o número de frutos e de árvores necessário para estimar a produção de sementes (kg) de uma árvore ou da população (n), foi determinado em função da variabilidade, do erro de amostragem admitido e da probabilidade de confiança fixada, conforme Péllico Netto \& Brena (1997): $\mathrm{n}=\mathrm{Nt}^{2} \mathrm{~s}_{\mathrm{t}}^{2} / \mathrm{NE}^{2}+\mathrm{t}^{2} \mathrm{~s}_{\mathrm{x}}^{2}$, em que $\mathrm{s}_{\mathrm{x}}^{2}$ é a estimativa da variância (g por fruto); $\mathrm{N}$ é o número total de frutos; E é o erro de amostragem admitido; e t é o valor de $t$ de Student.

$\mathrm{O}$ erro de amostragem admitido no inventário foi fixado a partir do limite percentual da média estimada, e calculado para a probabilidade de $95 \%$ com o limite de erro de 1,5 e $10 \%$, para o número de frutos por árvore, e 10,15 e $20 \%$, para o número de indivíduos na população: $\mathrm{E}^{2}=(\mathrm{LE} \times \overline{\mathrm{x}})^{2}$, em que LE é o limite do erro de amostragem admitido (1, 5 e $10 \%)$; $\bar{x}$ é a média (g por fruto).

Como as varáveis diâmetro à altura do peito (DAP), tipo morfológico, posição, forma, área e presença de cipós na copa se correlacionam significativamente com a produção de frutos e sementes da castanheira-do-brasil em florestas naturais (Wadt et al., 2005; Braga, 2007; Kainer et al., 2007; Ivanov, 2011), estas variáveis foram consideradas quanto aos possíveis ganhos de precisão na pré-estratificação da população. A viabilidade da estratificação pela variável de interesse - no caso, a produção de sementes (pós-estratificação) - também foi estudada, tendo-se verificado o padrão de produção da população e a proporção de indivíduos que permaneceram em um mesmo estrato, durante os anos de estudo.

Para a comparação entre dois grupos (tipo morfológico), empregou-se o teste t. Uma vez que o número de árvores com produção nula aumenta a variância e, consequentemente, o tamanho da amostra e os custos de inventário, procurou-se estudar a sua ocorrência em função da classe de diâmetro e da posição e forma da copa.

Nas amostras estratificadas, a distribuição ou alocação das árvores no estrato foi realizada de forma proporcional ao número de árvores no estrato $\left(\mathrm{n}_{\mathrm{h}}=\mathrm{n} \times \mathrm{N}_{\mathrm{h}} / \mathrm{N}\right)$ ou à variação na produção de sementes. Neste caso, utilizou-se a alocação proposta por Neyman (1964), em que se consideram custos iguais de amostragem em todos os estratos: $\mathrm{n}_{\mathrm{h}}=\mathrm{n} \times \mathrm{W}_{\mathrm{h}} \mathrm{s}_{\mathrm{h}} \sum^{\mathrm{L}} \mathrm{W}_{\mathrm{h}} \mathrm{s}_{\mathrm{h}}$, em que $\mathrm{n}$ é o tamanho da amostra; $\mathrm{N}$ é o número total de árvores na população; $\mathrm{n}_{\mathrm{h}}$ é o número de árvores amostradas no estrato $h ; N_{h}$ é o número total de árvores no estrato $\mathrm{h} ; \mathrm{W}_{\mathrm{h}}$ é a proporção do estrato $h$ na população; $S_{h}$ é o desvio-padrão da amostra no estrato h; e L é o número de estratos.

Em relação à periodicidade, as árvores-amostras foram sorteadas, em cada ano, de forma independente (amostragem temporária), ou sorteadas apenas no primeiro ano e mantidas nos anos subsequentes (amostragem contínua). Com base na relação entre as características da árvore (DAP, tipo morfológico e variáveis de copa), o tipo de alocação e a periodicidade da amostra, foram testados os seguintes procedimentos de amostragem: aleatória simples temporária; aleatória simples contínua; temporária estratificada pelo DAP, com alocação proporcional ao número de árvores por classe diamétrica; similar ao terceiro procedimento, porém sem amostrar árvores com má formação de copa e em posições inferiores do dossel (amostragem casual); estratificada pelo DAP contínua, com alocação proporcional ao número de árvores por classe diamétrica; estratificada casual contínua; e casual temporária, com alocação ótima.

Para verificar a precisão e a acurácia da amostragem, e definir o número de estratos, foram tomadas 16 amostras (quatro por ano) para cada procedimento de amostragem testado, o que totalizou 128 amostras na população. A intensidade de amostragem utilizada foi a de $20 \%$ do número de árvores da população. O número de estratos foi determinado conforme o método proposto por Péllico Netto \& Sanqueta (1995), que é definido a partir da razão entre a precisão calculada com a amostragem estratificada e aquela obtida para a amostragem aleatória irrestrita, quando esta tende a ser assintótica. Assim, o número de estratos a ser aplicado à população $(\mathrm{L})$ foi obtido por $\mathrm{Q}_{\mathrm{L}}=\mathrm{s}_{\mathrm{x} \text { est }}^{2} / \mathrm{s}_{\mathrm{x} \text { aleat, }}^{2}$ em que $\mathrm{s}_{\mathrm{x} \text { est }}^{2}$ é a variância da média estratificada; e $\mathrm{s}_{\mathrm{x} \text { aleat }}^{2}$ é a variância da média para a amostragem inteiramente aleatória. A relação $\mathrm{Q}_{\mathrm{L}}$ e o número de estratos foram obtidos pela função $\mathrm{Q}_{\mathrm{L}}=\mathrm{b}_{0}+\mathrm{b}_{1} \times 1 / \mathrm{L}$, em que $\mathrm{b}_{0} \mathrm{e}$ $\mathrm{b}_{1}$ são os coeficientes do modelo; e L é o número de estratos

Após a determinação do número de estratos, procedeu-se à análise de variância da estratificação, conforme Péllico Netto \& Sanqueta (1995). O número de estratos testados variou de 2 a 10. 
A precisão do inventário foi medida com base no intervalo de confiança para a média, no erro-padrão da média e no erro absoluto.

A acurácia foi medida pelo erro absoluto do inventário (Er), que representa a medida da diferença entre a média do parâmetro e a média estimada pela amostragem e é determinado por $\mathrm{E}_{\mathrm{r}}=100\left(\overline{\mathrm{x}}_{\mathrm{r}}-\overline{\mathrm{x}}_{\mathrm{e}}\right) / \overline{\mathrm{x}}_{\mathrm{r}}$, em que $\overline{\mathrm{x}}_{\mathrm{r}}$ é a média paramétrica; e $\overline{\mathrm{x}}_{\mathrm{e}}$ é a média estimada.

\section{Resultados e Discussão}

Apenas no ano de 2008 foram identificadas diferenças significativas de produção por tipo morfológico $\left(\mathrm{T}_{(92)}=2,505 ; \mathrm{p}=0,014\right)$. A proporção do número de indivíduos na população e a produção média de sementes, observada nos quatro anos foram de $58,03 \%$ e $3,34 \mathrm{~kg}$, em castanheiras vermelhas, e de $41,9 \%$ e $4,87 \mathrm{~kg}$, em brancas. Este resultado está parcialmente de acordo com os obtidos por Braga (2007), que observou diferenças significativas na produção acumulada de frutos (5 anos) entre 133 castanheiras vermelhas e brancas, em Epitaciolândia, AC. No entanto, no estudo de Braga (2007), as castanheiras vermelhas apresentaram maior produção de frutos, com frutos maiores, mais pesados e com menor número de sementes, o que não ocorreu na área estudada em Roraima.

A correlação entre DAP e a produção de sementes foi significativa, porém baixa $(r=0,204 ; p=0,001)$. Pode-se observar (Figura 1) que as maiores produções de sementes foram obtidas nas classes intermediárias, entre 50 a $150 \mathrm{~cm}$. A percentagem de árvores com $\mathrm{DAP} \leq 50 \mathrm{~cm}$ que produziram frutos foi baixa, e variou de 0 a $21,7 \%$ entre os anos.

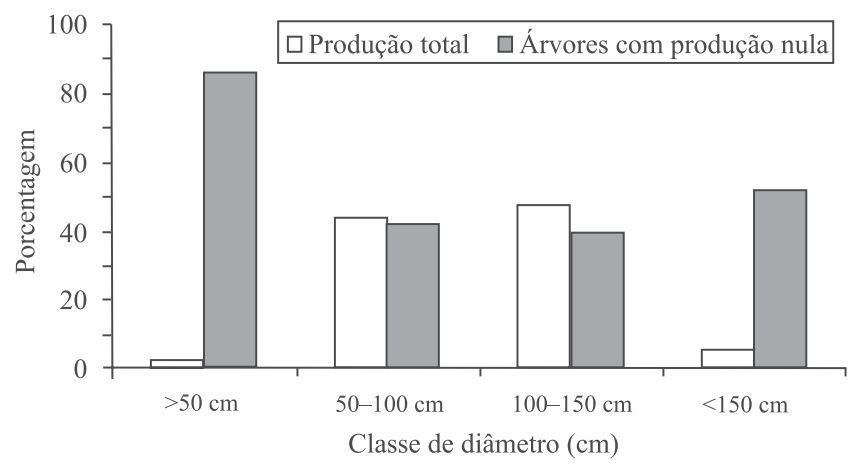

Figura 1. Relação entre as classes diamétricas e a participação na produção total.
Apesar do percentual de árvores que produziram frutos ter sido elevado $(69,5$ a $100 \%)$, as árvores com DAP superior a $150 \mathrm{~cm}$ apresentaram menor produção e contribuíram pouco para a produção total (com $5,49 \%$ ), mas muito para o número de árvores com produção nula (52\%). A participação de árvores com diâmetros inferiores a $50 \mathrm{~cm}$ na produção foi em média de apenas 2,62\%, com um alto percentual de árvores com produção nula $(86,4 \%)$. As árvores com metade ou menos da copa também contribuíram muito pouco para a produção $(4,7 \%)$ (Tabela 1$)$.

Observou-se que as árvores que ocupam as posições superiores no dossel foram responsáveis por $95,2 \%$ da produção. Nas classes diamétricas inferiores $(\mathrm{DAP} \leq 50 \mathrm{~cm})$, a percentagem de indivíduos com produção nula foi sempre elevada (acima de $82 \%$ ), independentemente da posição da copa (Figura 2). As árvores com diâmetro entre $50 \mathrm{~cm}$ e $100 \mathrm{~cm}$, que ocupavam posições subdossel, apresentaram elevada percentagem de indivíduos com produção nula $(68,4 \%)$ e contribuíram pouco para a produção total. A opção de utilizar uma amostragem casual e de não amostrar árvores com copas incompletas, localizadas nas posições inferiores do dossel, foram confirmadas com estes resultados. A expectativa foi de reduzir a variância, o esforço amostral, os custos de amostragem e aumentar a precisão do inventário florestal.

$\mathrm{Na}$ análise estatística dos 513 frutos (Tabela 2), observou-se DAP médio de 10,29 $\pm 1,52 \mathrm{~cm}$, com amplitude de 16,30 a $7,18 \mathrm{~cm}$. O número médio de sementes por fruto foi de $15,00 \pm 3,47$, com máximo de 24 e mínimo de 14, o que está dentro da variação observada por Zuidema \& Boot (1992), em diferentes castanhais na Amazônia, com valores máximos de 21 a 26 e mínimos de 7 a 13 sementes por fruto. $O$ diâmetro dos frutos foi ligeiramente inferior ao observado por

Tabela 1. Produção total de sementes $(\mathrm{kg})$ por características da copa.

\begin{tabular}{lc}
\hline Característica da copa & Produção total $(\mathrm{kg})$ \\
\hline Posição & \\
Emergente & $1.251,81$ \\
Dossel & 485,85 \\
Subdossel & 87,83 \\
\hline Forma & \\
Completa circular & $1.203,60$ \\
Completa irregular & 536,20 \\
Meia copa & 76,70 \\
Pobre & 9,26 \\
\hline
\end{tabular}


Santos et al. (2006), $(\mathrm{n}=1)$ de $10,7 \mathrm{~cm}$, e o número de sementes por fruto foi inferior ao observado por Camargo et al. (2010), com 15,17 a 19,86 sementes por fruto, em plantio localizado em Cotriguaçu, MT.

A massa média de sementes frescas por fruto foi de $114,43 \pm 45,23 \mathrm{~g}$, com o máximo de $264,34 \mathrm{~g}$ e o mínimo de $24,70 \mathrm{~g}$. Identificou-se correlação positiva e significativa entre a massa de sementes e o diâmetro médio dos frutos $(\mathrm{r}=0,55, \mathrm{p}=0,001)$.

Com base na amplitude diamétrica, os frutos foram classificados em três classes de tamanho: pequenos, de 7,18 a 10,22 cm; médios, de 10,22 a 13,34 cm; e grandes, maiores do que 13,34 cm. Observou-se predominância de frutos pequenos e médios $(95,36 \%)$ na produção. Apenas três árvores apresentaram frutos grandes, e uma apresentou predominância de frutos médios e grandes, o que é indicação de que a estratificação por tamanho de fruto deve ser realizada por árvore e torna a estratificação por este critério desaconselhável, por ser lenta e onerosa. No entanto, para plantios de enriquecimento e para futuros programas de melhoramento, deve-se procurar selecionar árvores de frutos grandes, com diâmetros iguais ou superiores a $13,34 \mathrm{~cm}$.

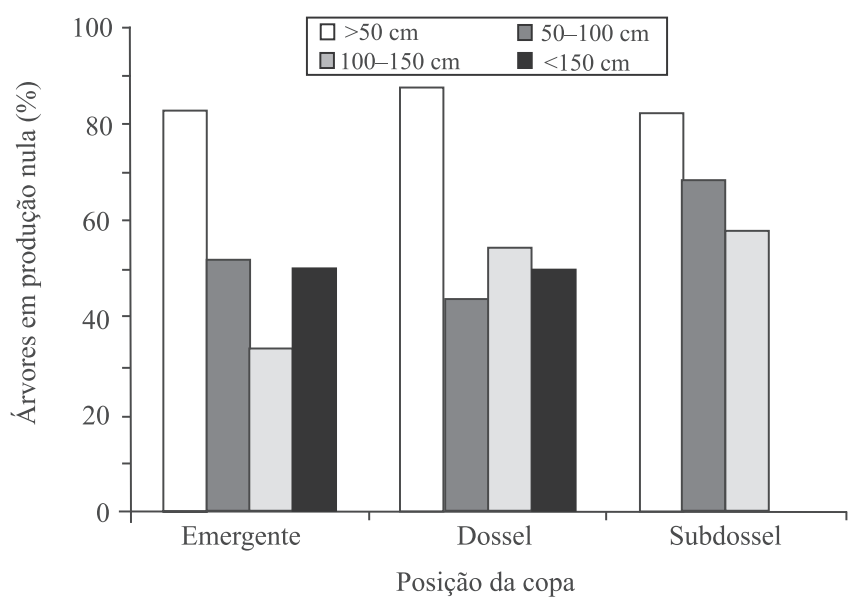

Figura 2. Relação entre a classe diamétrica, a posição da copa e a percentagem de árvores com produção nula.

Tabela 2. Valores médios para 513 frutos de oito árvores de castanheira-do-brasil, coletados durante o ano de 2010.

\begin{tabular}{lcccc}
\hline Variável & Média & Desvio-padrão & Máximo & Mínimo \\
\hline Diâmetro $(\mathrm{cm})$ & 10,29 & 1,52 & 16,30 & 7,18 \\
№ de sementes & 14,94 & 3,47 & 24,00 & 14,00 \\
Peso de sementes & 114,43 & 45,23 & 264,34 & 24,68 \\
\hline
\end{tabular}

O cálculo do tamanho da amostra (Figura 3) indicou que, para um limite de erro de $1 \%$ da média, foi necessário amostrar, em média, 97,3\% dos frutos, com variação entre 93,4 e 100\%. Para o limite de erro de $5 \%$, o tamanho da amostra foi, em média, de $64,6 \%$, com variação entre 41 e $68,7 \%$ e, para o limite de erro de $10 \%$, o tamanho da amostra foi de $33 \%$, com variação entre 15,3 e $48,4 \%$. Portanto, para serem obtidas estimativas precisas de produção ( $1 \%$ de erro), é necessário amostrar praticamente todos os frutos, e para estimar a produtividade de uma determinada árvore, com até $10 \%$ de erro, podese reduzir a amostragem para, em média, 33\% do número de frutos. Contudo, o tamanho da amostra deve, preferencialmente, ser definido com base na variabilidade individual na população, pois amostras pequenas podem resultar em erros de estimativa. Este deve ser o objeto de futuros estudos

Menores reduções no valor do $\mathrm{Q}_{\mathrm{L}}$ foram observadas a partir de seis estratos (Figura 4), para a amostra completa, e de quatro estratos, para a amostra casual, sem que tenham sido amostradas árvores com má formação de copa e em posições inferiores no dossel. A análise de variância para a estratificação (Tabela 3) indicou que não houve diferença significativa entre as médias dos estratos, em todos os anos, o que indica que a estratificação pelo DAP não foi plenamente satisfatória, uma vez que, para ser eficiente, seria desejável a ocorrência de diferenças significativas entre estratos e médias de produção, nos estratos, em todos os anos.

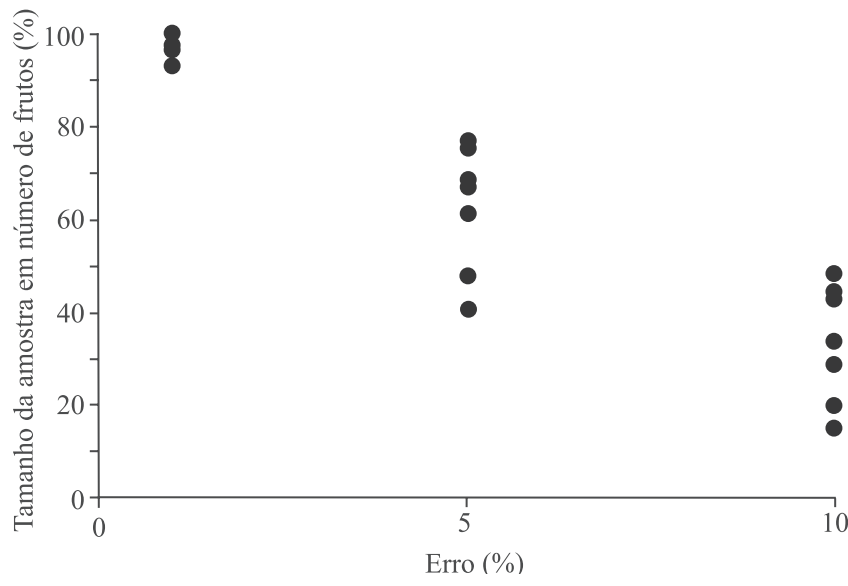

Figura 3. Tamanho da amostra de frutos de castanha-do-brasil, em função do limite de erro pré-fixado.

Pesq. agropec. bras., Brasília, v.48, n.5, p.519-527, maio 2013 DOI: 10.1590/S0100-204X2013000500008 
A estratificação pela variável de interesse (no caso, a produção de sementes) seria viável apenas se as árvores apresentassem tendência a permanecer no mesmo estrato, entre safras consecutivas. Para verificar esta condição, as árvores foram agrupadas em cinco classes de produção (nula, baixa, média, alta, e super), de acordo com a amplitude de variação na produção de sementes em determinado ano. Os limites de classe foram obtidos com a divisão da amplitude pelo número de classes desejado. Estas classes permitiram observar o padrão de produção da população (Tabela 4).

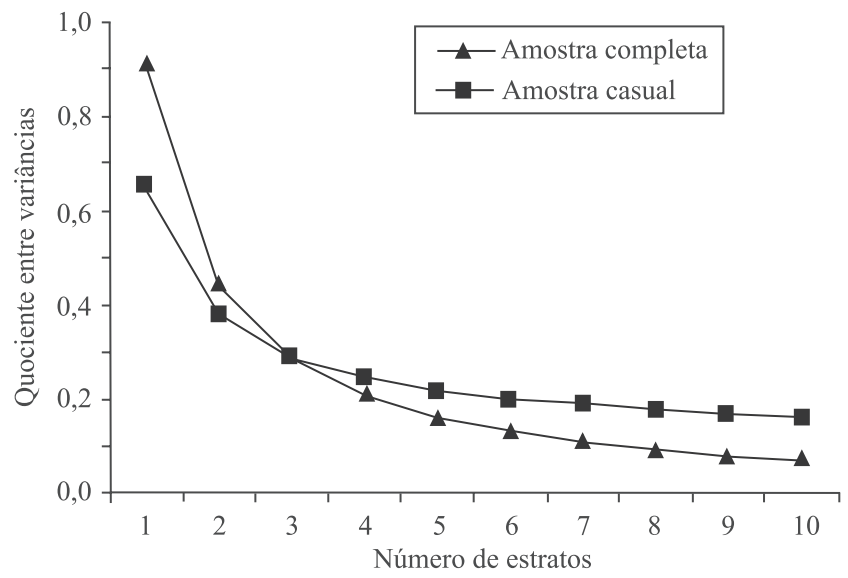

Figura 4. Relação entre a redução relativa do quociente entre variâncias $\left(\mathrm{Q}_{\mathrm{L}}\right)$ e o numero de estratos necessários para estimar a produção de frutos $(\mathrm{kg})$ em castanhal nativo.
A grande maioria das árvores $(59,3 \%)$ alternou anos de produção nula e baixa, e a sua participação na produção total foi elevada, em razão do grande número de indivíduos. A maior participação na produção total foi observada em árvores que alternaram anos de alta e baixa produção $(29,2 \%)$. Apenas 47 árvores permaneceram no mesmo estrato durante os quatro anos - 29 árvores de produção sempre nula e 18 de produção baixa -, o que inviabilizou a pós-estratificação com base na produção de sementes, para a população. A probabilidade de que uma árvore de alta produção, no primeiro monitoramento, voltasse a ter grande produção em quatro anos foi de $31,5 \%$. Esta probabilidade dobra ao se estender o período para dois anos de monitoramento $(66,6 \%)$, o que indica que pós-estratificações, com base na produção de sementes, devem ser feitas apenas após longos períodos de monitoramento.

Em um tamanho de amostra de $20 \%$ do número de árvores, o erro-padrão da estimativa e os erros médios absolutos variaram de 0,27 a 0,45 e de 22,16 a 49,92\%, respectivamente (Tabela 5). As amostras aleatórias (1 e 2) foram precisas e acuradas, tendo sido a temporária mais precisa e a contínua mais acurada. No entanto, no caso da amostra do tipo 1, os limites do intervalo de confiança (95\%), apesar de estreitos, não incluíram a verdadeira média da população dos anos de 2007 e 2010 (Tabela 6), o que é uma indicação de que, na intensidade amostral estudada ( $20 \%$ do número

Tabela 3. Análise de variância para a estratificação, em função da produção e classe diamétrica nas amostras completa e casual.

\begin{tabular}{|c|c|c|c|c|c|c|}
\hline Ano & Método & Fonte de variação & Graus de liberdade & Quadrado médio & F (calculado) & $\mathrm{p}$ \\
\hline \multirow[t]{2}{*}{2007} & \multirow{2}{*}{$\begin{array}{c}\text { Amostra } \\
\text { completa }\end{array}$} & Entre estratos & 5 & 128,99 & \multirow[t]{2}{*}{3,55} & \multirow[t]{2}{*}{0,01} \\
\hline & & Dentro dos estratos & 176 & 41,46 & & \\
\hline \multirow[t]{2}{*}{2008} & \multirow{2}{*}{$\begin{array}{l}\text { Amostra } \\
\text { completa }\end{array}$} & Entre estratos & 5 & 8,47 & \multirow[t]{2}{*}{1,95} & \multirow[t]{2}{*}{0,088} \\
\hline & & Dentro dos estratos & 176 & 4,33 & & \\
\hline \multirow[t]{2}{*}{2009} & \multirow{2}{*}{$\begin{array}{l}\text { Amostra } \\
\text { completa }\end{array}$} & Entre estratos & 5 & 82,67 & \multirow[t]{2}{*}{2,68} & \multirow[t]{2}{*}{0,023} \\
\hline & & Dentro dos estratos & 176 & 30,82 & & \\
\hline \multirow[t]{2}{*}{2010} & \multirow{2}{*}{$\begin{array}{l}\text { Amostra } \\
\text { completa }\end{array}$} & Entre estratos & 5 & 47,72 & \multirow[t]{2}{*}{2,87} & \multirow[t]{2}{*}{0,016} \\
\hline & & Dentro dos estratos & 176 & 16,57 & & \\
\hline \multirow[t]{2}{*}{2007} & \multirow{2}{*}{$\begin{array}{c}\text { Amostra } \\
\text { casual }\end{array}$} & Entre estratos & 3 & 75,96 & \multirow[t]{2}{*}{1,53} & \multirow[t]{2}{*}{0,21} \\
\hline & & Dentro dos estratos & 118 & 49,64 & & \\
\hline \multirow[t]{2}{*}{2008} & \multirow{2}{*}{$\begin{array}{c}\text { Amostra } \\
\text { casual }\end{array}$} & Entre estratos & 3 & 13,65 & \multirow[t]{2}{*}{2,34} & \multirow[t]{2}{*}{0,077} \\
\hline & & Dentro dos estratos & 118 & 5,82 & & \\
\hline \multirow[t]{2}{*}{2009} & \multirow{2}{*}{$\begin{array}{c}\text { Amostra } \\
\text { casual }\end{array}$} & Entre estratos & 3 & 102,40 & \multirow[t]{2}{*}{2,78} & \multirow[t]{2}{*}{0,044} \\
\hline & & Dentro dos estratos & 118 & 36,77 & & \\
\hline \multirow[t]{2}{*}{2010} & \multirow{2}{*}{$\begin{array}{c}\text { Amostra } \\
\text { casual }\end{array}$} & Entre estratos & 3 & 4,67 & \multirow[t]{2}{*}{2,19} & \multirow[t]{2}{*}{0,883} \\
\hline & & Dentro dos estratos & 118 & 21,38 & & \\
\hline
\end{tabular}

p, probabilidade para o valor de F. 
de árvores), amostras aleatórias não são recomendadas para esta população. Para o limite de $10 \%$ de erro, seria necessário amostrar $68 \%$ das árvores.

Em comparação à aleatória, as amostras estratificadas temporárias, com amostragem casual com alocação proporcional ou ótima (4 e 7), promoveram maior homogeneidade de variância e, consequentemente, redução do tamanho da amostra para um mesmo erro de amostragem admitido, com precisão e acurácia próximas. No entanto, os limites para o intervalo de confiança da média estimada (Tabela 6), em alguns anos, foram muito amplos, o que indica baixa precisão. Entre as amostras permanentes $(2,5$ e 6$)$, a menos precisa foi a do tipo 6 e a mais acurada e precisa foi a do tipo 5 . A amostragem do tipo contínua estratificada pelo DAP propiciou melhores estimativas do que a temporária.

A partir do princípio de que um método de amostragem deve ser definido de forma a ser estatisticamente confiável, representativo e eficiente no uso dos recursos (o que depende da relação entre o numero de árvores amostradas e do coeficiente de variação), o melhor procedimento de amostragem foi

Tabela 4. Padrão de produção de árvores de castanheira-do-brasil, em quatro anos de observação.

\begin{tabular}{lcc}
\hline $\begin{array}{l}\text { Classe de } \\
\text { produção }\end{array}$ & $\begin{array}{c}\text { Percentagem } \\
\text { de árvores }\end{array}$ & $\begin{array}{c}\text { Percentagem } \\
\text { na produção }\end{array}$ \\
\hline Nula & 15,9 & 0,0 \\
Nula e baixa & 59,3 & 26,4 \\
Alta & 0,0 & 0,0 \\
Alta ou média & 2,2 & 11,6 \\
Média ou baixa & 6,6 & 12,9 \\
Alta ou baixa & 6,6 & 29,2 \\
Alta ou nula & 4,4 & 13,1 \\
Demais & 4,4 & 6,9 \\
\hline
\end{tabular}

o tipo 5, pois, para um mesmo limite de erro admitido, ele permitiu um menor tamanho de amostra, com boa precisão e acurácia.

Amostragens contínuas têm a vantagem de garantir o monitoramento do indivíduo ao longo do tempo, possibilitam maior facilidade de localização das árvores, e são as mais utilizadas para o desenvolvimento de modelos de crescimento e produção. No entanto, tais amostras não podem ser consideradas independentes, o que é pré-requisito para a maioria dos testes estatísticos convencionais. A falta de dependência aumenta ou reduz o valor da probabilidade e o poder estatístico a graus desconhecidos (Gotelli \& Ellison, 2011). A independência das amostras só é garantida se a opção for por procedimentos aleatórios independentes, como o procedimento de amostragem 3. Na amostragem aleatória não estratificada, não se pode garantir que todas as classes diamétricas estejam representadas na amostra, e o DAP, além de ser de fácil obtenção no campo, é uma importante variável preditora na estimativa de produção para a espécie (Kainer et al., 2007). A alocação ótima, apesar de reduzir o coeficiente de variação e, consequentemente, o tamanho da amostra, apresenta a desvantagem de necessitar de um conhecimento prévio da variância dos estratos. Com este tipo de locação, ocorreram casos de superamostragem e até mesmo de não amostragem em alguns estratos, o que não é desejável, pois se perde parte das informações, sem ganhos na acurácia da amostra.

Reduzir o tamanho do universo amostral, sem amostrar árvores com má formação de copa, localizadas nas posições inferiores do dossel, não permitiu ganhos de precisão e acurácia, o que decorreu da produção inexpressiva destas árvores e da baixa correlação da produção com o DAP.

Tabela 5. Estatísticas de amostragem e tamanho da amostra para os procedimentos amostrais testados.

\begin{tabular}{|c|c|c|c|c|c|c|c|c|c|}
\hline \multirow[t]{2}{*}{ Amostragem $^{(1)}$} & \multirow{2}{*}{$\begin{array}{l}\text { Número de } \\
\text { amostras }\end{array}$} & \multirow[t]{2}{*}{ Média } & \multirow[t]{2}{*}{ Variância } & \multirow{2}{*}{$\begin{array}{l}\mathrm{CV} \\
(\%)\end{array}$} & \multirow[t]{2}{*}{$\mathrm{S}_{\mathrm{x}}$} & \multirow{2}{*}{$\begin{array}{c}\text { Erro } \\
\text { absoluto }\end{array}$} & \multicolumn{3}{|c|}{ Tamanho da amostra $^{(2)}$} \\
\hline & & & & & & & $10 \%$ & $15 \%$ & $20 \%$ \\
\hline 1 & 16 & 2,07 & 16,96 & 177.32 & 0,27 & 23,67 & $161(67,6)$ & $142(59,7)$ & $122(51,3)$ \\
\hline 2 & 16 & 2,39 & 25,09 & 182,35 & 0,32 & 27,75 & - & - & - \\
\hline 3 & 16 & 2,55 & 28,43 & 209,09 & 0,36 & 25,41 & $117(49,1)$ & $101(42,4)$ & $89(37,4)$ \\
\hline 4 & 16 & 2,60 & 19,56 & 170,10 & 0,36 & 39,81 & $84(35,3)$ & $72(30,2)$ & $72(30,2)$ \\
\hline 5 & 16 & 2,40 & 21,71 & 194,95 & 0,30 & 22,16 & - & - & - \\
\hline 6 & 16 & 2,93 & 32,04 & 193,18 & 0,45 & 49,92 & - & - & - \\
\hline 7 & 16 & 2,98 & 22,53 & 158,90 & 0,39 & 36,80 & $80(33,6)$ & $69(28,9)$ & $61(25,6)$ \\
\hline
\end{tabular}

(1)Procedimento de amostragem 1, aleatória temporária; 2, aleatória contínua; 3, estratificada pelo DAP, temporária com alocação proporcional; 4, estratificada pelo DAP, casual, temporária com alocação proporcional; 5, estratificada pelo DAP, contínua com alocação proporcional; 6 , estratificada pelo DAP, casual, contínua com alocação proporcional; 7, estratificada pelo DAP, casual, temporária com alocação ótima. ${ }^{(2)}$ Tamanho da amostra para 10,15 e $20 \%$ de erro. $S_{x}$, erro-padrão de estimativa. 
Tabela 6. Intervalo de confiança para a média estimada com os procedimentos amostrais testados.

\begin{tabular}{|c|c|c|c|c|}
\hline \multirow[t]{2}{*}{ Ano } & \multirow[t]{2}{*}{$\begin{array}{l}\text { Média } \\
(\mathrm{kg})\end{array}$} & \multirow[t]{2}{*}{ Amostragem $^{(1)}$} & \multicolumn{2}{|c|}{$\begin{array}{l}\text { Intervalo de confiança para } \\
\text { a média estimada }(95 \%)\end{array}$} \\
\hline & & & Mínimo & Máximo \\
\hline \multirow{7}{*}{2007} & \multirow{7}{*}{4,41} & 1 & 3,58 & 4,40 \\
\hline & & 2 & 2,85 & 5,76 \\
\hline & & 3 & 2,47 & 5,95 \\
\hline & & 4 & 1,57 & 5,87 \\
\hline & & 5 & 3,89 & 6,11 \\
\hline & & 6 & 2,64 & 6,32 \\
\hline & & 7 & 2,79 & 8,62 \\
\hline \multirow{7}{*}{2008} & \multirow{7}{*}{0,84} & 1 & 0,32 & 1,25 \\
\hline & & 2 & 0,27 & 1,47 \\
\hline & & 3 & 0,43 & 1,44 \\
\hline & & 4 & 0,79 & 1,19 \\
\hline & & 5 & 0,24 & 1,25 \\
\hline & & 6 & 0,12 & 2,57 \\
\hline & & 7 & 0,22 & 2,53 \\
\hline \multirow{7}{*}{2009} & \multirow{7}{*}{2,80} & 1 & 1,33 & 3,44 \\
\hline & & 2 & 1,01 & 4,67 \\
\hline & & 3 & 1,38 & 4,5 \\
\hline & & 4 & 2,05 & 4,65 \\
\hline & & 5 & 1,73 & 3,13 \\
\hline & & 6 & 0,69 & 6,46 \\
\hline & & 7 & 1,58 & 5,08 \\
\hline \multirow{7}{*}{2010} & \multirow{7}{*}{1,77} & 1 & 0,58 & 1,67 \\
\hline & & 2 & 0,46 & 2,60 \\
\hline & & 3 & 1,32 & 2,97 \\
\hline & & 4 & 1,55 & 6,20 \\
\hline & & 5 & 0,51 & 2,31 \\
\hline & & 6 & 1,46 & 6,67 \\
\hline & & 7 & 1,27 & 2,11 \\
\hline
\end{tabular}

(1)Procedimento de amostragem 1, aleatória temporária; 2, aleatória contínua; 3, estratificada pelo DAP, temporária com alocação proporcional; 4, estratificada pelo DAP, casual, temporária com alocação proporcional; 5, estratificada pelo DAP, contínua com alocação proporcional; 6 , estratificada pelo DAP, casual, contínua com alocação proporcional; 7, estratificada pelo DAP, casual, temporária com alocação ótima.

\section{Conclusões}

1. Estimativas precisas da produção de sementes em árvores de castanheira-do-brasil são obtidas em grandes amostras (em média, 97\% dos frutos); para erros de até $10 \%$, pode-se reduzir a amostragem para, em média, $33 \%$ do número de frutos.

2. A retirada de amostras contínuas, estratificadas pelo DAP em seis estratos, e a alocação proporcional ao número de árvores na classe diamétrica são os procedimentos mais indicados para amostragem.

\section{Agradecimentos}

Ao Conselho Nacional de Desenvolvimento Científico e Tecnológico (CNPq), pelo auxílio financeiro; aos senhores Adebaldo Sampaio Teles, José de Anchieta Moreira da Costa e Taiguara dos Santos Pereira, pelo apoio na coleta dos dados; e a José Lopes Primo, proprietário da fazenda Pau-Rainha, onde se realizaram os estudos.

\section{Referências}

ALECHANDRE, A.; BROWN, F.; CAMPOS, C.A.; AZEVEDO, K.; MELO, T. Cartilha de mapeamento de espécies florestais: um guia simplificado para a sistematização em planilha eletrônica de mapa de campo de espécies florestais. Rio Branco: Universidade Federal do Acre, 2007. 22p.

BOOT, R.G.A. Extraction of non-timber forest products from tropical rain forest. Does diversity come at a price? Netherlands Journal of Agricultural Science, v.45, p.439-450, 1997.

BRAGA, E.T.M. Diversidade morfológica e produção de Bertholletia excelsa H.B.K (Lecythidaceae) no sudeste do Estado do Acre - Brasil. 2007. 54p. Dissertação (Mestrado) Universidade Federal do Acre, Rio Branco.

CAMARGO, F.F.; COSTA, R.B. da; RESENDE, M.D.V. de; ROA, R.A.R.; RODRIQUES, N.B.; SANTOS, L.V. dos; FREITAS, A.C.A. de. Variabilidade genética para caracteres morfométricos de matrizes de castanha-do-brasil da Amazônia mato-grossense. Acta Amazônica, v.40, p.705-710, 2010. DOI: 10.1590/ S0044-59672010000400010.

CAVALCANTI, F.J. de B.; MACHADO, S. do A.; HOSOKAWA, R.T. Tamanho de unidade de amostra e intensidade amostral para espécies comerciais da Amazônia. Floresta, v.39, p.207-214, 2009.

EVANS, T.D.; VIENGKHAM, O.V. Inventory time-cost and statistical power: a case study of a Lao rattan. Forest Ecology and Management, v.150, p.313-322, 2001. DOI: 10.1016/ S0378-1127(00)00589-2.

FARIAS, L.L. Tamanho e forma de parcela amostral para inventários de espécies não madeireiras da Amazônia central. 2012. 114p. Dissertação (Mestrado) - Instituto Nacional de Pesquisada Amazônia, Manaus.

GOTELLI, N.J.; ELLISON, A.M. Princípios de estatística em ecologia. Porto Alegre: Artmed, 2011. 528p.

HIGGINS, M.A.; RUOKOLAINEN, K. Rapid tropical forest inventory: a comparison of techniques based on inventory data from western Amazonia. Conservation Biology, v.18, p.799-811, 2004. DOI: 10.1111/j.1523-1739.2004.00235.x.

IVANOV, G.B. Influência de variáveis dendrométricas anatômicas e ambientais na produção de frutos e sementes de Bertholletia excelsa H.B.K. 2011. 93p. Dissertação (Mestrado) Universidade Federal de Santa Maria, Santa Maria.

KAINER, K.A.; WADT, L.H.O.; STAUDHAMMER, C.L. Explaining variation in Brazil nut fruit production. Forest Ecology 
and Management, v.250, p.244-255, 2007. DOI: 10.1016/j. foreco.2007.05.024.

NEYMAN, J. On the two different aspects of the representative method: the method of stratified sampling and the method of purposive selection. Journal of the Royal Statistical Society, v.97, p.558-625, 1964.

PÉLLICO NETO, S.; BRENA, D.A. Inventário florestal. Curitiba: Ed. S. Péllico Netto, D.A. Brena, 1997. 316p.

PÉLLICO NETO, S.; SANQUETA, C.R. Determinação do número de estratos em estratificação volumétrica de florestas naturais e plantadas. Floresta, v.24, p.49-58, 1995.

PERES, C.A.; BAIDER, C.; ZUIDEMA, P.A.; WADT, L.H.O.; KAINER, K.A.; GOMES-SILVA, D.A.P.; SALOMÃO, R.P.; SIMÕES, L.L.; FRANCIOSI, E.R.N.; VALVERDE, F.C.; GRIBEL, R.; SHEPARD JUNIOR, G.H.; KANASHIRO, M.; COVENTRY, P.; YU, D.W.; WATKINSON, A.R.; FRECKLETON, R.P. Demographic threats to the sustainability of Brazil nut exploitation. Science, v.302, p.2112-2114, 2003. DOI: 10.1126/ science.1091698.

SALOMÃO, R.P. Densidade, estrutura e distribuição espacial de castanheira-do-brasil (Bertholletia excelsa H. \& B.) em dois platôs de floresta ombrófila densa na Amazônia setentrional brasileira.

Boletim do Museu Paraense Emilio Goeldi, v.4, p.11-25, 2009.

SANTOS, J.U.M. dos; BASTOS, M.N. do C.; GURGEL, E.S.C.; CARVALHO, A.C.M. Bertholletia excelsa Humboldt \& Bonpland (Lecythidaceae): aspectos morfológicos do fruto, da semente e da plântula. Boletim do Museu Paraense Emílio Goeldi, v.1, p.103-112, 2006.
SYNNOTT, T.J. A manual of permanent plot procedures for tropical rainforests. Oxford: University of Oxford, 1979. 67p. (University of Oxford-Commonwealth Forestry Institute. Tropical forestry papers, 14).

TONINI, H. Fenologia da castanheira-do-brasil (Bertholletia excelsa Humb. \& Bonpl., Lecythidaceae) no sul do Estado de Roraima. Cerne, v.17, p.123-131, 2011.

UBIALLI, J.A.; FIGUEIREDO FILHO, A.; MACHADO, S. do A.; ARCE, J.E. Comparação de métodos e processos de amostragem para estimar a área basal para grupos de espécies em uma floresta ecotonal da região norte matogrossense. Acta Amazonica, v.39, p.305-314, 2009. DOI: 10.1590/ S0044-59672009000200009.

WADT, L.H.O.; KAINER, K.A.; GOMES-SILVA, D.A.P. Population structure and nut yield of a Bertholletia excelsa stand in southwestern Amazonia. Forest Ecology and Management, v.211, p.371-384, 2005. DOI: 10.1016/j.foreco.2005.02.061.

WONG, J.L.G.; THORNBER, K.; BAKET, N. Resource assessment of non-wood forest products: experience and biometric principles. Rome: FAO, 2001. 110p. (FAO. Non-wood forest products technical papers, 13).

ZUIDEMA, P.A.; BOOT, R.G.A. Demography of the Brazil nut tree (Bertholletia excelsa) in the Bolivian Amazon: impact of seed extraction on recruitment and population dynamics. Journal of Tropical Ecology, v.18, p.1-31, 2002. DOI: 10.1017/ S0266467402002018.

Recebido em 16 de novembro de 2012 e aprovado em 15 de abril de 2013 